\title{
Factors Associated with Hepatitis B and C Co-Infection among HIV-Infected Patients in Singapore, 2006-2017
}

\author{
Chiaw Yee Choy ${ }^{1,2}$, Li Wei Ang ${ }^{1,3}{ }^{10}$, Oon Tek $\mathrm{Ng}^{1,2,4}$, Yee Sin Leo ${ }^{1,2,4,5}$ and \\ Chen Seong Wong $1,2,5, *$
}

1 National Centre for Infectious Diseases, 16 Jalan Tan Tock Seng, Singapore 308422, Singapore; chiawyee.choy@mohh.com.sg (C.Y.C.); li_wei_ang@ncid.sg (L.W.A.); oon_tek_ng@ttsh.com.sg (O.T.N.); yee_sin_leo@ncid.sg (Y.S.L.)

2 Department of Infectious Diseases, Tan Tock Seng Hospital, 11 Jalan Tan Tock Seng, Singapore 308433, Singapore

3 Public Health Group, Ministry of Health, Singapore, College of Medicine Building, 16 College Road, Singapore 169854, Singapore

4 Lee Kong Chian School of Medicine, Nanyang Technological University, 11 Mandalay Road, Singapore 308232, Singapore

5 Yong Loo Lin School of Medicine, National University of Singapore, 10 Medical Drive, Singapore 117597, Singapore

* Correspondence: chen_seong_wong@ttsh.com.sg; Tel.: +65-6357-7415

Received: 26 February 2019; Accepted: 24 May 2019; Published: 27 May 2019

\begin{abstract}
Co-infection of hepatitis B virus (HBV) and hepatitis C virus (HCV) with human immunodeficiency virus (HIV) is associated with increased risk of hepatic complications and mortality. A retrospective study to estimate the proportion of HBV and HCV co-infections in Singapore was conducted using a clinical database. We included 3065 patients who were seen under the Clinical HIV Programme at the largest referral centre for HIV care between 2006 and 2017 and were tested for both HBV and HCV. Factors associated with HIV-HBV and HIV-HCV co-infections were determined using logistic regressions. The majority (86.3\%) of HIV-infected patients were mono-infected, while $7.2 \%$ were co-infected with $\mathrm{HBV}, 6.0 \%$ with $\mathrm{HCV}$, and $0.5 \%$ were co-infected with both $\mathrm{HBV}$ and $\mathrm{HCV}$. The most common HCV genotype was GT1 (63\%). Factors significantly associated with HBV co-infection in the multivariable model were: Aged 30-49 years and 50-69 years at HIV diagnosis, male gender, and HIV transmission through intravenous drug use (IDU). Independent factors associated with HCV co-infection were: Malay ethnicity, HIV transmission through IDU, and HIV diagnosis between 2006 and 2008. Behavioural risk factors such as IDU, as well as epidemiologic differences associated with co-infection, should inform further studies and interventions aimed at reducing viral hepatitis infection among HIV-infected individuals.
\end{abstract}

Keywords: HIV; hepatitis; HBV; HCV; co-infection; prevalence

\section{Introduction}

Human immunodeficiency virus (HIV), hepatitis B virus (HBV), and hepatitis C virus (HCV) are the most common chronic viral infections worldwide [1]. These three viruses share common modes of transmission, which include sexual transmission, sharing of needles with intravenous drug use (IDU), and transfusion of blood and blood products. Patients can often be co-infected with two, and even all three, viruses. 
Prior to the availability of highly active antiretroviral therapy (HAART), patients with HIV-HBV co-infection were 19 times more likely than patients mono-infected with HBV to die from liver-related complications [2]. The advent of tenofovir, lamivudine, and emtricitabine have led to significant improvements in reducing hepatic complications and related mortality. However, despite these advances, some studies continue to show that overall mortality and risk of hepatocellular carcinoma remains elevated in HIV-HBV co-infected populations compared to HIV or HBV mono-infected populations [3,4], while others have shown better prognosis with the advent of HAART [5].

Approximately 37 million people worldwide are infected with HIV, and about $7.4 \%$ of them are co-infected with HBV [6]. The estimated prevalence of HBV infection worldwide varies across different geographical regions [7]. In Singapore, the prevalence of HBV was $0.3 \%$ in children and adolescents aged 1-17 years between 2008 and 2010 [8], and 3.6\% in adults aged 18-79 years in 2010 [9]. The reduction in the incidence of acute hepatitis $B$ and the prevalence of chronic hepatitis $B$ carriage in Singapore has been attributed to the introduction of hepatitis $B$ vaccination into the national childhood immunisation programme (NCIP) in 1987 [10].

The estimated prevalence of $\mathrm{HCV}$ infection worldwide is $2.2 \%$ [11], while the estimated global prevalence of $\mathrm{HCV}$ co-infection among people living with $\mathrm{HIV}$ is 6.2\% [12]. As of 2016, the prevalence of $\mathrm{HCV}$ in Singapore is estimated to be about $0.1 \%$ based on blood donor screening data and notifications of new HCV cases to the Ministry of Health (MOH) [13].

In Singapore, about 450 new cases of HIV are reported every year, and this number has remained fairly constant since 2008, with notification rates of 103.7 to 125.2 per million resident population [14]. The majority (97\%) of newly-reported HIV infections from 2008-2017 were acquired through sexual transmission [14].

A local study investigating the risk factors and time trends for co-infections in a random sample of over 700 HIV-infected patients who were referred for first-time care from 2006 to 2011 found that $8.1 \%$ were co-infected with $\mathrm{HBV}$ and $2 \%$ had active $\mathrm{HCV}$ co-infection [15]. It is important to understand risks contributing to co-infection to guide public health interventions and surveillance. In this retrospective study, we investigated the epidemiological factors associated with HBV and HCV co-infection among HIV-infected patients in Singapore.

\section{Materials and Methods}

\subsection{Study Population}

The Clinical HIV Programme at the National Centre for Infectious Diseases (NCID) sees the highest number of HIV-infected patients in Singapore. The NCID is a 330-bed purpose-built facility and houses clinical services, public health, research, training, and education under one overarching structure. We made use of a clinical database maintained by the programme for the retrospective study, with the following information being routinely collected for all patients referred for care: Demographics, virologic and immunologic parameters, antiretroviral therapy (ART) and monitoring, and routine biochemical test results performed in the course of HIV care.

The study population comprised Singapore residents who were diagnosed with HIV and attended the NCID at least once between 2006 and 2017. We confined the study subjects to HIV-infected patients who were tested for both HBV and HCV. HIV infection was confirmed by western blot assay following a positive result from a fourth-generation chemiluminescent microparticle immunoassay (CMIA). HBV infection was ascertained based on seropositivity for the hepatitis B antigen surface (HBsAg) using electrochemiluminescence immunoassay (ECLIA), and hepatitis B e-antigen (HBeAg) was also measured using ECLIA. HCV infection was based on seropositivity for the anti-HCV antibody (anti-HCV) test using ECLIA, or detectable HCV with real-time polymerase chain reaction (PCR). HCV genotyping was done by reverse line probe hybridisation.

Co-infection means an affected person is living with two or more infections at the same time. Individuals should not be considered as having both HIV and HBV and/or HCV infections 
simultaneously at any time point if they have been cleared of HBV or HCV prior to their HIV diagnosis. Approximately $95 \%$ of adults newly infected with HBV recover completely and do not become chronically infected $[16,17]$. After acute $\mathrm{HCV}$ infection, up to $45 \%$ of individuals achieve spontaneous viral clearance within six months of infection without any treatment, and do not progress to chronic infection $[18,19]$. Hence, patients with positive results for HBV based on HBsAg and/or HCV based on anti-HCV or HCV PCR, more than one year before being tested positive for HIV, were excluded, as a large number of these patients may have been cleared of HBV and/or HCV prior to their HIV diagnosis. We checked the subsequent test results of patients excluded in this way, and they were found to be negative for HBV/HCV. In addition, we would like to investigate risk factors associated with $\mathrm{HIV}$ and $\mathrm{HBV} / \mathrm{HCV}$ co-infections. HBV or HCV infection acquired before HIV infection may have been due to risk factors that are different from HIV infection, hence they were excluded from the data analysis. Serologic test results up to the end of September 2018 were retrieved from the clinical database. Co-infection with HBV was defined as a positive test for HBsAg within one year before HIV diagnosis, or a positive HBsAg result after HIV diagnosis. Co-infection with $\mathrm{HCV}$ was defined as a positive test for anti-HCV and/or HCV PCR within one year before HIV diagnosis, or a positive HCV test after HIV diagnosis.

Epidemiological characteristics and laboratory parameters were compared in four groups of HIV-infected patients: HIV mono-infection, co-infections with HBV only, co-infections with HCV only, and HIV-HBV-HCV co-infections.

\subsection{Statistical Methods}

We calculated the $95 \%$ confidence intervals (CIs) for binomial proportions using Wilson's method. The Chi-square test was used to test for group differences. Proportions between two groups were compared using two-sample independent $\mathrm{z}$ tests, with standard error estimated using pooled value of the two proportions. The Mantel-Haenszel linear-by-linear association Chi-square test was used to evaluate whether there was linear trend in proportion of co-infections over time.

The main outcomes of interest were whether the patient had HBV or HCV co-infections or not. For univariable logistic regression analyses, odds ratios (OR) and 95\% CIs were calculated. Multivariable logistic regression was used to determine independent risk factors associated with co-infections, and adjusted odds ratios (aOR) were calculated. Variables were considered for inclusion in the multivariable model using the likelihood-ratio statistic based on the maximum partial likelihood estimates in backward stepwise selection with $p>0.10$ for removal of variables. All variables with $p<0.05$ were retained in the final multivariable model.

The Kaplan-Meier method was used to estimate the five-year survival rate. All reports of deaths, irrespective of cause, which occurred by the end of 2017 were included in the survival analysis. Patients contributed person-time from the date of HIV diagnosis until death or 31 December 2017, whichever was the earlier date.

All $p$ values reported were two-sided, and statistical significance was taken as $p<0.05$. Statistical analyses were performed using the IBM SPSS Statistics for Windows, version 24 (IBM Corp., Armonk, NY, USA).

\subsection{Ethical Considerations}

Ethical approval for use of the clinical database was obtained from the Singapore National Healthcare Group Domain Specific Review Board (NHG DSRB reference number 2012/00438). Informed consent was not obtained as the clinical data collected was used as part of the care management of HIV patients. All data analysed for the study were anonymised.

\section{Results}

A total of 3327 HIV-infected patients were seen under the Clinical HIV Programme from 2006 to 2017. There were 3139 patients who had been tested for HBV, and among them, 3097 had also been 
tested for HCV. After excluding 32 who had been tested positive for $\mathrm{HBV}$ and/or HCV more than one year before HIV diagnosis and subsequently tested negative, a total of 3065 HIV-infected patients constituted the final sample for this study. The proportion of HBV co-infection among HIV-infected patients was $7.7 \%$ (95\% CI: 6.8-8.7\%), while the proportion with HCV co-infection was $6.6 \%(95 \%$ CI: $5.7-7.5 \%)$. The majority $(86.3 \%)$ were mono-infected, while $0.5 \%$ were co-infected with HBV and HCV.

The mean age at HIV diagnosis was 40.2 years (standard deviation 12.7; range: 13 to 88). Adults aged $20-49$ years at HIV diagnosis comprised about $74.3 \%$ of the study sample. The majority $(93.8 \%)$ were men, and over three-quarters $(76.0 \%)$ were Chinese. The main mode of HIV transmission was via sexual exposure (96.6\%). Most of the HIV-infected patients (95.5\%) were on antiretroviral treatment at the time of analysis.

Patients with triple infections of HIV, HBV, and HCV were diagnosed with HIV at a younger age (mean age 36.8, median age 35); the majority (87.6\%) were aged $20-49$ years at diagnosis (Table 1 ). About three-quarters of patients with HIV mono-infection, and those co-infected with HCV only, were in the age group of $20-49$ years; $73.8 \%$ in HIV mono-infected patients (mean age 40.2 , median age 39 ), and $74.6 \%$ in HIV-infected patients co-infected with HCV only (mean age 38.4, median age 38). Patients who were co-infected with HBV only were diagnosed with HIV at an older age (mean age 43.1, median age 41 ), and $70.5 \%$ were aged $20-49$ years at time of HIV diagnosis.

In the HIV mono-infected group, $93.3 \%$ were men-a significantly lower proportion compared to $97.7 \%$ in those co-infected with HBV only $(p=0.01)$ (Table 1). Patients of Chinese ethnicity constituted $85.0 \%$ of those co-infected with HBV only, and this proportion was significantly higher than that of the HIV mono-infected group $(76.2 \%)(p=0.003)$ and the group who were co-infected with HCV only $(63.2 \%)(p<0.005)$. Patients of Malay ethnicity comprised $30.3 \%$ of those co-infected with HCV only, and this proportion was significantly higher than that in the HIV mono-infected group (15.1\%) and in the group who were co-infected with HBV only $(10.0 \%)$ (both $p<0.0005)$.

Across the four groups, the majority were infected via sexual mode of transmission (Table 1). Among patients co-infected with HCV only, 30.2\% were infected through IDU—a significantly higher proportion compared to $2.2 \%$ in the HIV mono-infected group and $3.7 \%$ in those co-infected with HBV only (both $p<0.0005$ ).

A significantly higher proportion of patients co-infected with HCV (40.0\%) were diagnosed with HIV in the period 2006-2008, compared to $28.3 \%$ in the mono-infected group ( $p=0.01$ ) (Table 1 ). In the two groups of patients co-infected with $\mathrm{HCV}$ only and those with triple infection, about one-third had used recreational or illicit drugs, while the proportion with unknown history of drug use was $50 \%$ or higher.

Among patients co-infected with $\mathrm{HBV}$ only, about $57.3 \%$ had both infections diagnosed within one month (Table 1). Among patients co-infected with HCV only, a significantly lower proportion of $30.2 \%$ had both infections diagnosed within one month, compared to those co-infected with HBV only $(p<0.0005)$. About one-quarter of patients co-infected with HCV only $(24.9 \%)$ tested positive for HCV more than 48 months after HIV diagnosis. In the group with triple infections, about $37.5 \%$ had HIV and either HBV and/or HCV diagnosed within one month.

There was a significant decline in the proportion of HIV-infected patients who were co-infected with HBV over the period of HIV diagnosis; from 8.8\% in 2006-2008 (95\% CI: 7.1-10.8\%) to 5.5\% in 2015-2017 (95\% CI: 4.0-7.7\%) (test for trend, $p=0.037$ ) (Figure 1). The proportion of HIV-infected patients who were co-infected with HCV also saw a significant decrease; from $9.0 \%$ in $2006-2008$ (95\% CI: 7.3-11.0\%) to $4.5 \%$ in 2015-2017 (95\% CI: 3.1-6.5\%) (test for trend, $p=0.003$ ). 


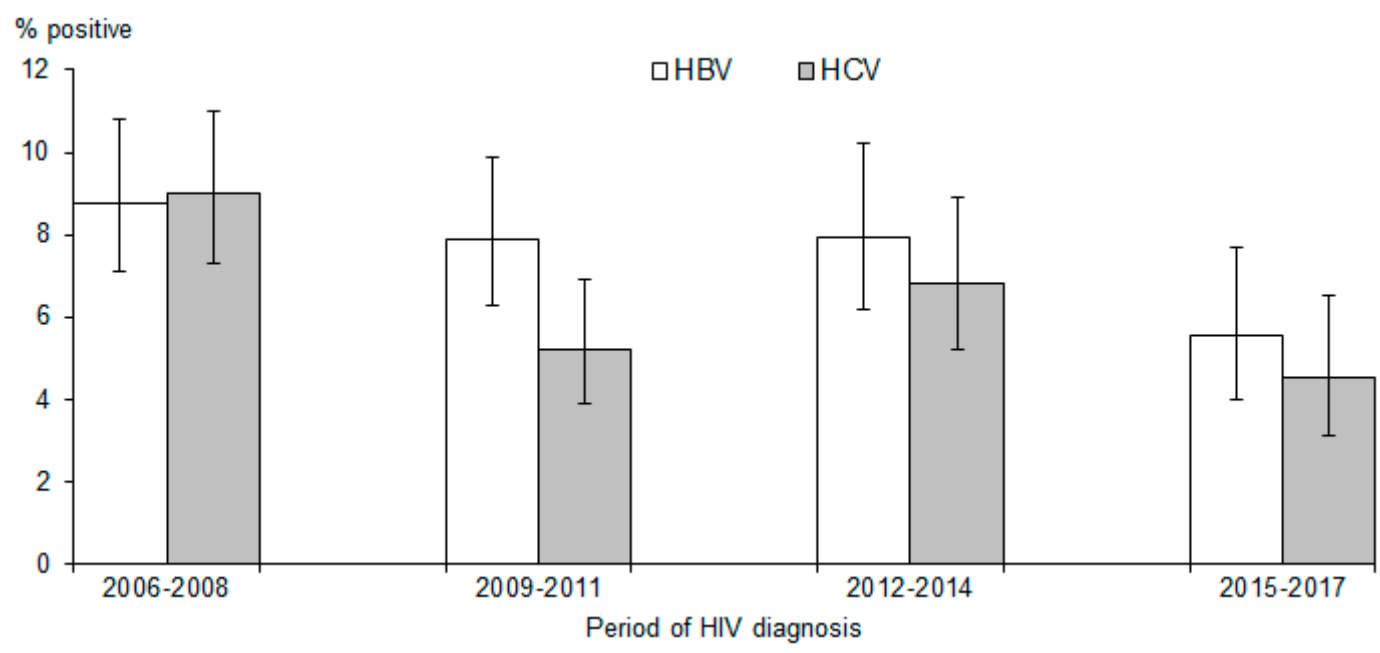

Figure 1. Proportions of HIV-infected patients tested positive for HBV and HCV by period of HIV diagnosis. The vertical lines indicate $95 \%$ confidence intervals.

Among patients with CD4 measured at HIV diagnosis, over half (56.3\%) of those co-infected with HBV only had CD4 count $\leq 200$ cells $/ \mathrm{mm}^{3}$, which was significantly higher compared to $46.1 \%$ in the HIV mono-infected group $(p=0.005)$ and $39.8 \%$ in those co-infected with HCV only $(p=0.002)$ (Table 2$)$. Among patients with aspartate aminotransferase (AST) measured at HIV diagnosis, about $26.3 \%$ of those co-infected with HBV only had AST $>48 \mathrm{U} /$ liter, which was significantly higher compared to $11.6 \%$ in the HIV mono-infected group $(p<0.0005)$. Among patients with alanine aminotransferase (ALT) measured at HIV diagnosis, about $21.4 \%$ of those co-infected with HBV only had ALT $>55 \mathrm{U} /$ liter, which was significantly higher compared to $10.5 \%$ in the HIV mono-infected group $(p<0.0005)$.

Of the $236 \mathrm{HIV}$ patients co-infected with HBV, 216 (91.5\%) underwent tenofovir-containing ART. Among the 180 patients tested positive for HBsAg who were also tested for HBeAg during the corresponding period, 85 (47.2\%, 95\% CI: 40.1-54.5\%) were positive for $\mathrm{HBeAg}$.

Univariable logistic regression analyses indicated that relative to HIV mono-infected patients, the factors associated with the presence of HBV co-infection were: Being in the age groups of 30-49 and 50-69 years, male gender, Chinese ethnicity, IDU as the mode of HIV transmission, diagnosis of HIV being made in the period 2006-2008, being diagnosed with AIDS-defining illnesses/opportunistic infections within one year of HIV diagnosis, and history of using recreational or illicit drugs (Table 3).

Multivariable logistic regression identified age group, gender, and mode of HIV transmission as significant factors that were independently associated with HBV co-infections among HIV-infected patients. Compared to patients diagnosed at 10-29 years of age, those who were diagnosed with HIV at 30-49 years (aOR 2.24, 95\% CI: 1.49-3.39) and 50-69 years (aOR 2.33, 95\% CI: $1.48-3.67$ ) were at higher odds of HBV co-infections. The odds of HBV co-infections were significantly higher among men than women (aOR 2.84, 95\% CI: 1.24-6.51). Compared to patients infected via sexual mode of transmission, those who were infected via IDU had over four times the odds of HBV co-infections (aOR 4.50, 95\% CI: 1.41-14.38).

Factors associated with the presence of $\mathrm{HCV}$ co-infections in univariable logistic regression analyses were: Malay ethnicity, IDU as the mode of HIV transmission, diagnosis of HIV in the period 2006-2008, and history of using recreational or illicit drugs (Table 4). In multivariable logistic regression, ethnic group, mode of HIV transmission, and period of HIV diagnosis were found to be independently associated with HCV co-infection. Malay patients had about twice the odds of HCV infection (aOR 2.19, 95\% CI: 1.10-4.34) compared with those of Indian or other ethnic minority. Compared to patients infected via sexual mode of transmission, those who were infected via IDU had nearly 20 times the odds of HCV co-infections (aOR 19.15, 95\% CI: 6.74-54.38). The odds of HCV co-infections in patients diagnosed with HIV in 2006-2008 were about two times (aOR 2.00, 95\% CI: 1.25-3.21) that of those diagnosed in 2015-2017. 
Table 1. Epidemiological characteristics of HIV-infected patients with mono-infection and HBV and HCV co-infections who attended the national referral centre for HIV in Singapore, 2006-2017.

\begin{tabular}{|c|c|c|c|c|c|c|}
\hline & $\begin{array}{l}\text { HIV } \\
\text { Mono-Infected } \\
n(\%)\end{array}$ & $\begin{array}{l}\text { Co-Infected with } \\
\text { HBV Only } \\
n(\%)\end{array}$ & $\begin{array}{l}\text { Co-Infected with } \\
\text { HCV Only } \\
n(\%)\end{array}$ & $\begin{array}{l}\text { Co-Infected with both } \\
\text { HBV and HCV } \\
n(\%)\end{array}$ & $p$ Value $^{+}$ & $\begin{array}{l}\text { All } \\
n(\%)\end{array}$ \\
\hline All & $2644(100.0)$ & $220(100.0)$ & $185(100.0)$ & $16(100.0)$ & & $3065(100.0)$ \\
\hline Age group (years) at HIV diagnosis & & & & & 0.004 & \\
\hline $10-19$ & $48(1.8)$ & $1(0.5)$ & $7(3.8)$ & $0(0.0)$ & & $56(1.8)$ \\
\hline $20-29$ & $578(21.9)$ & $23(10.5)$ & $39(21.1)$ & $5(31.3)$ & & $645(21.0)$ \\
\hline 30-39 & $718(27.2)$ & $72(32.7)$ & $59(31.9)$ & $5(31.3)$ & & $854(27.9)$ \\
\hline $40-49$ & $654(24.7)$ & $60(27.3)$ & 40 (21.6) & $4(25.0)$ & & $758(24.7)$ \\
\hline 50-59 & $454(17.2)$ & $42(19.1)$ & 34 (18.4) & $0(0.0)$ & & $530(17.3)$ \\
\hline $60-69$ & $149(5.6)$ & $19(8.6)$ & $6(3.2)$ & $2(12.5)$ & & $176(5.7)$ \\
\hline $70+$ & $43(1.6)$ & $3(1.4)$ & $0(0.0)$ & $0(0.0)$ & & $46(1.5)$ \\
\hline Gender & & & & & 0.032 & \\
\hline Female & $177(6.7)$ & $5(2.3)$ & $7(3.8)$ & $1(6.3)$ & & $190(6.2)$ \\
\hline Male & $2467(93.3)$ & $215(97.7)$ & $178(96.2)$ & $15(93.8)$ & & $2875(93.8)$ \\
\hline Ethnic Group & & & & & $<0.0005$ & \\
\hline Chinese & $2014(76.2)$ & $187(85.0)$ & $117(63.2)$ & $11(68.8)$ & & $2329(76.0)$ \\
\hline Malay & $400(15.1)$ & $22(10.0)$ & $56(30.3)$ & $5(31.3)$ & & $483(15.8)$ \\
\hline Indian & $138(5.2)$ & $2(0.9)$ & $4(2.2)$ & $0(0.0)$ & & $144(4.7)$ \\
\hline Others & $92(3.5)$ & $9(4.1)$ & $8(4.3)$ & $0(0.0)$ & & $109(3.6)$ \\
\hline Marital Status & & & & & 0.622 & \\
\hline Never married & $1431(54.1)$ & $116(52.7)$ & $96(51.9)$ & $10(62.5)$ & & $1653(53.9)$ \\
\hline Married & $591(22.4)$ & $48(21.8)$ & $32(17.3)$ & $3(18.8)$ & & $674(22.0)$ \\
\hline Separated/Divorced/Widowed & $265(10.0)$ & $26(11.8)$ & 25 (13.5) & $1(6.3)$ & & $317(10.3)$ \\
\hline Unknown & $357(13.5)$ & $30(13.6)$ & $32(17.3)$ & $2(12.5)$ & & $421(13.7)$ \\
\hline Mode of HIV transmission & & & & & $<0.0005$ & \\
\hline Homosexual & $1009(38.2)$ & 79 (35.9) & $65(35.1)$ & $8(50.0)$ & & $1161(37.9)$ \\
\hline Heterosexual & 1197 (45.3) & 99 (45.0) & 35 (18.9) & $3(18.8)$ & & 1334 (43.5) \\
\hline Bisexual & $306(11.6)$ & 25 (11.4) & $24(13.0)$ & $3(18.8)$ & & $358(11.7)$ \\
\hline IDU & $4(0.2)$ & $3(1.4)$ & $8(4.3)$ & $1(6.3)$ & & $16(0.5)$ \\
\hline IDU and sexual & $53(2.0)$ & $5(2.3)$ & $48(25.9)$ & $1(6.3)$ & & $107(3.5)$ \\
\hline Others & $13(0.5)$ & $1(0.5)$ & $1(0.5)$ & $0(0.0)$ & & $15(0.5)$ \\
\hline Unknown & $62(2.3)$ & $8(3.6)$ & $4(2.2)$ & $0(0.0)$ & & $74(2.4)$ \\
\hline Period of HIV diagnosis & & & & & 0.011 & \\
\hline 2006-2008 & $748(28.3)$ & $72(32.7)$ & $74(40.0)$ & $7(43.8)$ & & 901 (29.4) \\
\hline 2009-2011 & 755 (28.6) & $63(28.6)$ & 40 (21.6) & $5(31.3)$ & & $863(28.2)$ \\
\hline 2012-2014 & $605(22.9)$ & $53(24.1)$ & $45(24.3)$ & $3(18.8)$ & & $706(23.0)$ \\
\hline 2015-2017 & $536(20.3)$ & $32(14.5)$ & $26(14.1)$ & $1(6.3)$ & & 595 (19.4) \\
\hline
\end{tabular}


Table 1. Cont.

\begin{tabular}{|c|c|c|c|c|c|c|}
\hline & $\begin{array}{l}\text { HIV } \\
\text { Mono-Infected } \\
n(\%)\end{array}$ & $\begin{array}{l}\text { Co-Infected with } \\
\text { HBV Only } \\
n(\%)\end{array}$ & $\begin{array}{l}\text { Co-Infected with } \\
\text { HCV Only } \\
n(\%)\end{array}$ & $\begin{array}{l}\text { Co-Infected with both } \\
\text { HBV and HCV } \\
n(\%)\end{array}$ & $p$ Value $^{+}$ & $\begin{array}{l}\text { All } \\
n(\%)\end{array}$ \\
\hline $\begin{array}{l}\text { AIDS-defining illnesses/opportunistic } \\
\text { infections within one year of HIV diagnosis }\end{array}$ & & & & & 0.113 & \\
\hline No & $1396(52.8)$ & $98(44.5)$ & $92(49.7)$ & $8(50.0)$ & & $1594(52.0)$ \\
\hline Yes & $1248(47.2)$ & $122(55.5)$ & $93(50.3)$ & $8(50.0)$ & & $1471(48.0)$ \\
\hline Ever on ART & & & & & 0.620 & \\
\hline Yes & $2522(95.4)$ & 208 (94.5) & $182(98.4)$ & $16(100.0)$ & & $2928(95.5)$ \\
\hline No & $122(4.6)$ & $12(5.5)$ & $3(1.6)$ & $0(0.0)$ & & $137(4.5)$ \\
\hline Ever used recreational or illicit drugs * & & & & & $<0.0005$ & \\
\hline No & $542(20.5)$ & $34(15.5)$ & $33(17.8)$ & $0(0.0)$ & & $609(19.9)$ \\
\hline Yes & $251(9.5)$ & 27 (12.3) & $61(33.0)$ & $6(37.5)$ & & $345(11.3)$ \\
\hline Unknown & $1851(70.0)$ & $159(72.3)$ & $91(49.2)$ & $10(62.5)$ & & $2111(68.9)$ \\
\hline $\begin{array}{l}\text { Time first tested positive for HBV/HCV } \\
\text { Before HIV diagnosis }\end{array}$ & & & & & $<0.0005$ & \\
\hline 1-12 months & & $7(3.2)$ & $5(2.7)$ & $4(25.0)$ & & \\
\hline$<1$ month & & $23(10.5)$ & $11(5.9)$ & $2(12.5)$ & & \\
\hline From HIV diagnosis ** & & & & & & \\
\hline$<1$ month & & $103(46.8)$ & $45(24.3)$ & $4(25.0)$ & & \\
\hline $1-3$ months & & $53(24.1)$ & $34(18.4)$ & $3(18.8)$ & & \\
\hline 4-6 months & & $7(3.2)$ & $4(2.2)$ & $0(0.0)$ & & \\
\hline $7-12$ months & & $3(1.4)$ & $9(4.9)$ & $0(0.0)$ & & \\
\hline 13-24 months & & $5(2.3)$ & $13(7.0)$ & $2(12.5)$ & & \\
\hline $25-48$ months & & $10(4.5)$ & $18(9.7)$ & $1(6.3)$ & & \\
\hline$>48$ months & & $9(4.1)$ & $46(24.9)$ & $0(0.0)$ & & \\
\hline
\end{tabular}

${ }^{\dagger}$ Chi-square test was used to test for group differences. * Includes ecstasy, insufflated amyl nitrites or 'poppers', erectile dysfunction medications like sildenafil, amphetamines, cannabis, heroin, cocaine, barbiturates/ benzodiazepines, opium, psychedelic psilocybin mushrooms, solvents, LSD (Lysergic Acid Diethylamide). ${ }^{* *}$ Includes HIV-infected patients tested positive for $\mathrm{HBV}$ and/or HCV on the same day. 
Table 2. Baseline clinical characteristics of HIV-infected patients with mono-infection and HBV and HCV co-infections who attended the national referral centre for HIV in Singapore, 2006-2017 *.

\begin{tabular}{|c|c|c|c|c|c|c|}
\hline & $\begin{array}{l}\text { HIV Mono-Infected } \\
n(\%)\end{array}$ & $\begin{array}{l}\text { Co-Infected with } \\
\text { HBV Only } \\
n(\%)\end{array}$ & $\begin{array}{l}\text { Co-Infected with } \\
\text { HCV Only } \\
n(\%)\end{array}$ & $\begin{array}{l}\text { Co-Infected with both } \\
\text { HBV and HCV } \\
n(\%)\end{array}$ & $p$ Value $^{+}$ & $\begin{array}{l}\text { All } \\
n(\%)\end{array}$ \\
\hline CD4 (cells/mm³); $n(\%)$ & $2514(100.0)$ & $208(100.0)$ & $166(100.0)$ & $16(100.0)$ & 0.049 & $2904(100.0)$ \\
\hline$>350$ & $758(30.2)$ & $48(23.1)$ & $56(33.7)$ & $5(31.3)$ & & $867(29.9)$ \\
\hline $201-350$ & $598(23.8)$ & $43(20.7)$ & 44 (26.5) & $2(12.5)$ & & 687 (23.7) \\
\hline$\leq 200$ & $1158(46.1)$ & $117(56.3)$ & $66(39.8)$ & $9(56.3)$ & & $1350(46.5)$ \\
\hline Median CD4 [IQR] & 230 [53-392] & 143 [33-332] & $278[115-424]$ & 169 [22-393] & & 225 [53-390] \\
\hline HIV viral load (copies/mL); $n(\%)$ & $1689(100.0)$ & $141(100.0)$ & $112(100.0)$ & $12(100.0)$ & 0.212 & $1954(100.0)$ \\
\hline$\leq 200$ & $1618(95.8)$ & $135(95.7)$ & $107(95.5)$ & $10(83.3)$ & & $1870(95.7)$ \\
\hline$>200$ & $71(4.2)$ & $6(4.3)$ & $5(4.5)$ & $2(16.7)$ & & $84(4.3)$ \\
\hline Median viral load in $1000 \mathrm{~s}$ [IQR] & 83 [19-306] & 176 [33-559] & 83 [23-283] & $74[1-758]$ & & 87 [19-321] \\
\hline AST (U/liter); $n(\%)$ & $2021(100.0)$ & $179(100.0)$ & $125(100.0)$ & $12(100.0)$ & $<0.005$ & $2337(100.0)$ \\
\hline$\leq 48$ & 1787 (88.4) & $132(73.7)$ & $101(80.8)$ & $8(66.7)$ & & $2028(86.8)$ \\
\hline$>48$ & $234(11.6)$ & $47(26.3)$ & $24(19.2)$ & $4(33.3)$ & & 309 (13.2) \\
\hline Median AST (U/liter) [IQR] & 26 [21-35] & 32 [27-49] & 28 [23-38] & 35 [29-99] & & 27 [22-36] \\
\hline ALT (U/liter); $n(\%)$ & $2143(100.0)$ & $187(100.0)$ & $135(100.0)$ & $12(100.0)$ & $<0.005$ & $2477(100.0)$ \\
\hline$\leq 55$ & $1917(89.5)$ & $147(78.6)$ & $112(83.0)$ & $8(66.7)$ & & $2184(88.2)$ \\
\hline$>55$ & $226(10.5)$ & $40(21.4)$ & $23(17.0)$ & $4(33.3)$ & & $293(11.8)$ \\
\hline Median ALT (U/liter) [IQR] & $24[18-35]$ & $30[24-52]$ & 25 [17-39] & $36[28-82]$ & & $24[18-36]$ \\
\hline
\end{tabular}

${ }^{\dagger}$ Chi-square test was used to test for group differences. * Within \pm 6 months of HIV diagnosis. IQR, interquartile range. ALT, alanine transaminase; AST, aspartate transaminase. 
Table 3. Proportion and odds ratios of HBV co-infections in HIV-infected patients who attended the national referral centre for HIV in Singapore, $2006-2017$.

\begin{tabular}{|c|c|c|c|c|c|c|c|}
\hline & \multirow{2}{*}{$\begin{array}{l}\text { \% Co-Infected } \\
\text { with HBV }\end{array}$} & \multicolumn{3}{|c|}{ Univariable Model } & \multicolumn{3}{|c|}{ Multivariable Model ${ }^{* *}$} \\
\hline & & OR & $(95 \%$ CI $)$ & $p$ Value & aOR & $(95 \% \mathrm{CI})$ & $p$ Value \\
\hline Age at diagnosis (years) & & & & 0.001 & & & 0.001 \\
\hline $10-29$ & 4.1 & 1.00 & Referent & & 1.00 & Referent & \\
\hline $70+$ & 6.5 & 1.62 & $(0.47,5.52)$ & 0.443 & 1.77 & $(0.52,6.06)$ & 0.364 \\
\hline \multicolumn{8}{|l|}{ Gender } \\
\hline Female & 3.2 & 1.00 & Referent & & 1.00 & Referent & \\
\hline Chinese & 8.5 & 2.04 & $(1.10,3.81)$ & 0.024 & & & \\
\hline Malay & 5.6 & 1.30 & $(0.64,2.67)$ & 0.471 & & & \\
\hline Indian and others & 4.3 & 1.00 & Referent & & & & \\
\hline Marital status & & & & 0.953 & & & \\
\hline Single & 7.6 & 1.00 & Referent & & & & \\
\hline Married & 7.6 & 0.99 & $(0.71,1.39)$ & 0.963 & & & \\
\hline Divorced/Separated/Widowed & 8.5 & 1.13 & $(0.73,1.74)$ & 0.586 & & & \\
\hline Others and unknown & 10.1 & 1.37 & $(0.68,2.76)$ & 0.384 & 1.35 & $(0.67,2.74)$ & 0.404 \\
\hline Period of HIV diagnosis & & & & 0.148 & & & \\
\hline $2006-2008$ & 8.8 & 1.64 & $(1.08,2.49)$ & 0.022 & & & \\
\hline 2009-2011 & 7.9 & 1.46 & $(0.95,2.24)$ & 0.086 & & & \\
\hline 2012-2014 & 7.9 & 1.47 & $(0.94,2.29)$ & 0.091 & & & \\
\hline 2015-2017 & 5.5 & 1.00 & Referent & & & & \\
\hline \multicolumn{8}{|l|}{$\begin{array}{l}\text { AIDS-defining illnesses/opportunistic } \\
\text { infections within one year of HIV diagosis }\end{array}$} \\
\hline No & 6.6 & 1.00 & Referent & & & & \\
\hline Yes & 8.8 & 1.36 & $(1.04,1.78)$ & 0.024 & & & \\
\hline Ever used recreational or illicit drugs * & & & & 0.057 & & & \\
\hline No & 5.6 & 1.00 & Referent & & & & \\
\hline Yes & 9.6 & 1.79 & $(1.09,2.94)$ & 0.022 & & & \\
\hline Unknown & 8.0 & 1.47 & $(1.01,2.15)$ & 0.046 & & & \\
\hline
\end{tabular}

* Includes ecstasy, insufflated amyl nitrites or 'poppers', erectile dysfunction medications like sildenafil, amphetamines, cannabis, heroin, cocaine, barbiturates/ benzodiazepines, opium, psychedelic psilocybin mushrooms, solvents, LSD (Lysergic Acid Diethylamide). ${ }^{* *}$ Adjusted for age group, gender, and mode of HIV transmission. OR: Odds ratio. aOR: Adjusted odds ratio. 
Table 4. Proportion and odds ratios of HCV co-infections in HIV-infected patients who attended the national referral centre for HIV in Singapore, $2006-2017$.

\begin{tabular}{|c|c|c|c|c|c|c|c|c|}
\hline & & \multirow{2}{*}{$\begin{array}{l}\text { \% Co-Infected } \\
\text { with HCV }\end{array}$} & \multicolumn{3}{|c|}{ Univariable Model } & \multicolumn{3}{|c|}{ Multivariable Model ** } \\
\hline & & & OR & $(95 \% \mathrm{CI})$ & $p$ Value & $\mathrm{aOR}$ & $(95 \% \mathrm{CI})$ & $p$ Value \\
\hline \multicolumn{5}{|c|}{ Age at diagnosis (years) } & 0.800 & & & \\
\hline & $10-29$ & 7.3 & 1.00 & Referent & & & & \\
\hline & $30-49$ & 6.7 & 0.92 & $(0.65,1.29)$ & 0.615 & & & \\
\hline & $50-69$ & 5.9 & 0.81 & $(0.53,1.23)$ & 0.317 & & & \\
\hline & $70+$ & 0.0 & - & - & - & - & & \\
\hline \multicolumn{9}{|l|}{ Gender } \\
\hline & Female & 4.2 & 1.00 & Referent & & & & \\
\hline & Male & 6.7 & 1.64 & $(0.79,3.37)$ & 0.181 & & & \\
\hline \multirow[t]{4}{*}{ Ethnic group } & & & & & $<0.0005$ & & & 0.020 \\
\hline & Chinese & 5.5 & 1.17 & $(0.64,2.14)$ & 0.616 & 1.38 & $(0.72,2.62)$ & 0.330 \\
\hline & Malay & 12.6 & 2.90 & $(1.53,5.50)$ & 0.001 & 2.19 & $(1.10,4.34)$ & 0.026 \\
\hline & Indian and others & 4.7 & 1.00 & Referent & & 1.00 & Referent & \\
\hline \multirow[t]{5}{*}{ Marital status } & & & & & 0.169 & & & \\
\hline & Single & 6.4 & 1.00 & Referent & & & & \\
\hline & Married & 5.2 & 0.80 & $(0.54,1.18)$ & 0.264 & & & \\
\hline & Divorced/Separated/Widowed & 8.2 & 1.30 & $(0.83,2.04)$ & 0.244 & & & \\
\hline & Unknown & 8.1 & 1.28 & $(0.86,1.92)$ & 0.226 & & & \\
\hline \multicolumn{3}{|c|}{ Mode of HIV transmission } & & & $<0.0005$ & & & $<0.0005$ \\
\hline & Sexual & 4.8 & 1.00 & Referent & & 1.00 & Referent & \\
\hline & IDU & 56.3 & 25.30 & $(9.28,68.93)$ & $<0.0005$ & 19.15 & $(6.74,54.38)$ & $<0.0005$ \\
\hline & Sexual and IDU & 45.8 & 16.62 & $(10.95,25.22)$ & $<0.0005$ & 15.01 & $(9.69,23.25)$ & $<0.0005$ \\
\hline & Others and unknown & 5.6 & 1.17 & $(0.47,2.93)$ & 0.736 & 1.10 & $(0.44,2.76)$ & 0.845 \\
\hline \multicolumn{5}{|c|}{ Period of HIV diagnosis } & 0.002 & & & 0.004 \\
\hline & $2006-2008$ & 9.0 & 2.08 & $(1.33,3.25)$ & 0.001 & 2.00 & $(1.25,3.21)$ & 0.004 \\
\hline & 2009-2011 & 5.2 & 1.16 & $(0.71,1.89)$ & 0.558 & 1.06 & $(0.63,1.77)$ & 0.831 \\
\hline & 2012-2014 & 6.8 & 1.53 & $(0.95,2.49)$ & 0.083 & 1.51 & $(0.91,2.52)$ & 0.111 \\
\hline & 2015-2017 & 4.5 & 1.00 & Referent & & 1.00 & Referent & \\
\hline \multicolumn{9}{|c|}{ AIDS-defining illnesses/opportunistic infections within one year of HIV diagnosis } \\
\hline & No & 6.3 & 1.00 & Referent & & & & \\
\hline & Yes & 6.9 & 1.10 & $(0.83,1.47)$ & 0.508 & & & \\
\hline \multicolumn{5}{|c|}{ Ever used recreational or illicit drugs * } & $<0.0005$ & & & \\
\hline & No & 5.4 & 1.00 & Referent & & & & \\
\hline & Yes & 19.4 & 4.21 & $(2.71,6.54)$ & $<0.0005$ & & & \\
\hline & Unknown & 4.8 & 0.88 & $(0.59,1.31)$ & 0.524 & & & \\
\hline
\end{tabular}

* Includes ecstasy, insufflated amyl nitrites or 'poppers', erectile dysfunction medications like sildenafil, amphetamines, cannabis, heroin, cocaine, barbiturates/ benzodiazepines, opium, psychedelic psilocybin mushrooms, solvents, LSD (Lysergic Acid Diethylamide). ${ }^{* *}$ Adjusted for ethnic group, mode of HIV transmission, and period of HIV diagnosis. OR: Odds ratio. aOR: Adjusted odds ratio. 
Of the $201 \mathrm{HIV}$-infected patients co-infected with HCV within this study, 129 of them had HCV genotype testing. Excluding six with indeterminate genotype results, the common HCV genotype (GT) was GT-1 (63\%), followed by GT-3 (32\%) among 123 HIV-infected patients.

The cumulative proportion of patients in the HIV mono-infected group surviving until the fifth year since HIV diagnosis was $93 \%$, while it was $89 \%$ in those co-infected with HBV only and $91 \%$ in those co-infected with HCV only. There was no statistical difference in survival time between these three groups (log-rank test, $p=0.06$ ).

\section{Discussion}

The proportions of co-infections among HIV-infected patients in our study were 7.7\% for HIV-HBV co-infections, $6.0 \%$ for HIV-HCV co-infections, and $0.5 \%$ for HIV-HBV-HCV triple infections.

The proportion of HBV co-infections in this cohort is similar to countries of low endemicity, such as the United States (7.8\% to 8.6\%) [20] and Germany (9.4\%) [21]. In Southeast Asia, the prevalence of co-infection with HBV was estimated to be $10.4 \%$ [22]. When compared to specific neighbouring countries such as Malaysia (13\%) [23], Thailand (3.3\% to 13\%) [24], and Indonesia (3.2\% to $15.3 \%$ ) [24], the proportion of HIV-HBV co-infection in Singapore is lower.

The proportion of HCV co-infection among HIV-infected patients in this study was $6.0 \%$. In comparison, the overall prevalence of HIV-HCV co-infection in Western Europe and the United States was $25 \%$ to $30 \%$ [11] and $15.2 \%$ in Southeast Asia [22]. It is lower in Cambodia (5.5\%), Myanmar (5.3\%), and Thailand (5.1\%) [25], while Vietnam (42.5\%) and Indonesia (17.9\%) have higher prevalence of co-infection [25].

The significant risk factors associated with HIV-HBV co-infection in this study were being aged 30-49 years and 50-69 years at HIV diagnosis, male gender, and HIV transmission via IDU alone (Table 3). In Southeast Asia, a higher proportion of HIV-HBV co-infections were also seen mainly in those infected via IDU. Vietnam reported a co-infection rate of $28 \%$ in people who inject drugs (PWID) [26], while 20.1\% of Chinese PWID and 11.3\% of Burmese PWID in the China-Myanmar border region are HIV-HBV co-infected [27]. In Western Europe and the United States, the overall prevalence of HIV-HBV co-infection in PWID was 7\% to 10\% [11]. Higher prevalence of HBV co-infection was also noted in HIV-positive men having sex with men (MSM) in Western Europe and the United States (9\% to $17 \%$ ) [11]. This was not observed among the prevalence studies done in Southeast Asia [24]. In our study, $25 \%$ of patients who acquired HIV via IDU alone were co-infected with HBV (Table 3). As the absolute number of patients infected with HIV via IDU alone or IDU and sexual transmission who were also co-infected with HBV was much smaller than those who were co-infected with HCV (10 versus 58, Table 1), this suggests that IDU contributed more to the latter group as a risk factor.

Patients with HIV-HBV co-infection were diagnosed at an older age compared to those with HIV-HCV co-infections. There was a significant decline in the proportion of patients who were co-infected with HBV over the period of HIV diagnosis, from 8.8\% in 2006-2008 to 4.5\% in 2015-2017. These two findings could be attributed to the introduction of hepatitis $B$ vaccination as part of the NCIP; hepatitis B vaccination was introduced into the programme for babies born to hepatitis B-carrier mothers on 1 October 1985, and subsequently extended to all newborns on 1 September 1987 [28].

HIV transmission via IDU was also a significant risk factor independently associated with HIV-HCV co-infection in our study (Table 4). Our study revealed that the highest proportion of patients co-infected with HCV acquired HIV via IDU alone (56.3\%), followed by those infected with HIV via IDU and sexual mode of transmission (45.8\%) (Table 4). The proportion of HIV-HCV co-infection was $6.3 \%$ in patients infected with HIV via homosexual mode of transmission, $2.8 \%$ in those infected via heterosexual mode, and $7.5 \%$ in those who acquired HIV via bisexual mode (data not shown). In comparison, the prevalence of HIV-HCV co-infection in Western Europe and the United States has been reported to be affecting $72 \%$ to $95 \%$ of PWID, $1 \%$ to $12 \%$ of MSM, and $9 \%$ to $27 \%$ of heterosexuals [11]. Within the region, IDU and blood products were associated with increased risk of HIV-HCV co-infection, while female gender was a protective factor [22]. Similar high prevalence of 
HIV-HCV co-infections were seen in PWID: 31.8\% of Chinese and 23.9\% of Burmese PWID within the China-Myanmar border [27], and $89.8 \%$ to $98.5 \%$ in Vietnam [24]. As HCV is more efficiently transmitted via exposure to infected blood rather than sexual intercourse, the lower proportion of HIV-HCV co-infection in our study compared to the other European and Southeast Asia countries could be due to the relatively small number of PWID in Singapore who were infected with HIV via IDU.

Besides IDU as the mode of HIV transmission, Malay ethnicity and being diagnosed with HIV between 2006 and 2008 were also associated with HIV-HCV co-infections (Table 4). These results correspond to the pattern of drug use within Singapore. IDU is not common in Singapore, likely due to lack of supply and the deterrent effect of strict penalties for drug use and other related offences. In 2000, Subutex ${ }^{\circledR}$ (or buprenorphine hydrochloride) was approved by $\mathrm{MOH}$ as a substitute treatment for opiate-dependent drug abusers [29]. However, it was used inappropriately and injected as an intravenous cocktail mixed with other drugs. This unexpectedly resulted in a sudden increase in the number of PWID, where at least 3800 inappropriate users of Subutex were apprehended within four years of its introduction [29]. Subsequently, Subutex was made a controlled drug on 14 August 2006 [29]. As of the end of 2017, there were no new Subutex abusers reported [30]. The episode of Subutex abuse could have explained the higher HIV-HCV co-infection rate during 2006-2008 in this study. In addition, the highest number of drug abusers were found among persons of Malay ethnicity in 2017, which could explain the association seen in our study [30].

Patients with HIV-HCV co-infection were diagnosed at a younger age compared to HIV-HBV co-infection. The younger age seen in patients infected via IDU could be explained by the pattern of drug use in Singapore: About $64 \%$ of drug abusers arrested in 2017 were below the age of 30 years [30].

The most common HCV genotype seen in our study was GT-1, followed by GT-3. In a study on residual blood serum samples of blood donors screened between 2011 and 2014, 0.06\% were positive for HCV, and of the 42 serum samples available for genotyping, the distribution was $48 \%$ GT-3 and $31 \%$ GT-1 [31]. In Asia, GT-3 accounted for $40 \%$ of all HCV infection [32]. Regionally in Southeast Asia, GT-3 was the most common genotype seen in Thailand (44.2\%) and Malaysia (58.6\%), while GT-6 was more commonly seen in Cambodia (56\%), Laos (95.6\%), Myanmar (49\%), and Vietnam (54.5\%) [32].

There are limitations to this study. Our study is confined to patients followed-up at the NCID. However, as the NCID is the largest referral centre for HIV care, our study population could be considered as fairly representative of newly diagnosed HIV patients at large. Tests for HBV and HCV are mostly carried out on order from the treating physician, hence patients who did not have these two tests had to be excluded from the study. Treatment history was not available in the clinical database and we were unable to determine whether the HBV and/or HCV infection was chronic or acute. Patients with chronic HBV and/or HCV infection who had been tested positive more than one year prior to their HIV diagnosis could have been mis-classified as having acute infection and they were excluded from our study, but this number was expected to be small in view of the considerable proportion who would have achieved spontaneous viral clearance within six months of infection, particularly for HBV [16-19]. Moreover, all patients excluded in this way were further evaluated and found to have tested negative for $\mathrm{HBV} / \mathrm{HCV}$. Other variables such as socioeconomic status, educational level, and behavioural practices were not captured in the clinical database, but they may be potential risk factors of HBV and/or HCV co-infection. Information on other forms of hepatitis, such as hepatitis delta, was also not captured in the clinical database.

The proportion of viral hepatitis co-infections in patients living with HIV could be higher, but as Singapore follows the United States Department of Health and Human Services (DHHS) guidelines for management of HIV, almost all patients are screened for co-infections upon HIV diagnosis [33]. As part of the local practice, patients who are found to be non-immune are usually vaccinated against HBV, hence the number of subsequent HBV infections is likely to be small, as observed in this study. On the contrary, close to $25 \%$ of co-infection of $\mathrm{HCV}$ was detected more than 48 months after the diagnosis of HIV (Table 1). This problem of subsequent HCV infection following HIV infection is similar to that 
seen in other settings, and reflects the role of ongoing high-risk sexual and drug-using behaviour in driving HIV-HCV co-infection [34].

Given that IDU is the only modifiable risk factor for HIV-HCV co-infection in our study, it is important to consider interventions to control the spread of infections via this route. One of the key interventions that has been implemented in the United States and Europe to reduce the risk of transmission of HIV and other blood-borne viruses among PWID is harm reduction programmes, such as needle exchange programmes. Participation in these programmes was associated with reduction in the risk of HIV incidence by $33 \%$ in the United States, and by two to three folds in Amsterdam [35,36]. The non-use of syringe exchange was associated with a six-fold increased risk of HBV and a seven-fold increased risk of HCV in the United States [37]. In Amsterdam, full participation in the harm reduction programme resulted in a six- to seven-fold reduction in the risk of HCV seroconversion [36]. In Malaysia, since the implementation of the Needle and Syringe Exchange Programme in 2006, the percentage of HIV infections acquired via IDU has reduced from 75\% in 2006 to $47.7 \%$ in 2010 [38].

\section{Conclusions}

In conclusion, it is encouraging that there is a declining trend of co-infection with both HBV and $\mathrm{HCV}$ across the years, especially given that the number of new HIV diagnoses remains consistent since 2008. Many of the patients co-infected with HIV and HCV or triple infected were diagnosed with HIV at a younger age, reflecting the higher proportion of PWID seen in younger age groups. While the number of PWID in Singapore is low, it is still important to address the issue of IDU: Compared to patients infected with HIV via the sexual route, those who were infected via IDU had over four times the odds of HBV co-infections and 20 times the odds of HCV co-infections. In the absence of a HCV vaccine, other preventive interventions—such as risk-stratified screening, testing and treating, and behavioural interventions-are needed as part of hepatitis C control efforts in Singapore [39].

Author Contributions: Conceptualization, C.Y.C., L.W.A., C.S.W., and Y.S.L.; methodology, C.Y.C., L.W.A., and C.S.W.; software, L.W.A.; formal analysis, L.W.A.; investigation, O.T.N.; resources, O.T.N.; writing-original draft preparation, C.Y.C. and L.W.A.; writing-review and editing, C.Y.C., L.W.A., C.S.W., and Y.S.L.; visualization, C.Y.C. and L.W.A.; supervision, C.S.W. and Y.S.L.

Funding: This research received no external funding.

Acknowledgments: We thank the patients and staff in the NCID Clinical HIV Programme who made this study possible. Ng Oon Tek was supported by the NMRC Clinician Scientist Award (NMRC/CSA-INV/0002/2016).

Conflicts of Interest: The authors declare no conflict of interest.

\section{References}

1. Soriano, V.; Barreiro, P.; Nuñez, M. Management of Chronic Hepatitis B and C in HIV-Coinfected Patients. J. Antimicrob. Chemother. 2006, 57, 815-818. [CrossRef]

2. Singh, K.P.; Crane, M.; Audsley, J.; Avihingsanon, A.; Sasadeusz, J.; Lewin, S.R. HIV-Hepatitis B Virus Coinfection: Epidemiology, Pathogenesis, and Treatment. AIDS 2017, 31, 2035-2052. [CrossRef]

3. Pinchoff, J.; Tran, O.C.; Chen, L.; Bornschlegel, K.; Drobnik, A.; Kersanske, L.; Fuld, J. Impact of Hepatitis B on Mortality and Specific Causes of Death in Adults with and without HIV Co-Infection in NYC, 2000-2011. Epidemiol. Infect. 2016, 144, 3354-3364. [CrossRef]

4. Rajbhandari, R.; Jun, T.; Khalili, H.; Chung, R.T.; Ananthakrishnan, A.N. HBV/HIV Coinfection Is Associated with Poorer Outcomes in Hospitalized Patients with HBV or HIV. J. Viral Hepat. 2016, 23, 820-829. [CrossRef] [PubMed]

5. Piroth, L.; Pol, S.; Miailhes, P.; Lacombe, K.; Lopes, A.; Fillion, A.; Loustaud-Ratti, V.; Borsa-Lebas, F.; Salmon, D.; Rosenthal, E.; et al. Therapeutic Management and Evolution of Chronic Hepatitis B: Does HIV Still Have an Impact? The EPIB 2012 Study. Liver Int. 2015, 35, 1950-1958. [CrossRef]

6. UNAIDS. Miles To Go: Closing Gaps Breaking Barriers Righting Injustices. 2018. Available online: http://www.unaids.org/sites/default/files/media_asset/miles-to-go_en.pdf (accessed on 17 November 2018). 
7. WHO Hepatitis B Fact Sheets. Available online: https://www.who.int/news-room/fact-sheets/detail/hepatitis-b (accessed on 20 January 2019).

8. Ang, L.W.; Tey, S.H.; Cutter, J.; James, L.; Goh, K.T. Seroprevalence of hepatitis B virus infection among children and adolescents in Singapore, 2008-2010. J. Med. Virol. 2013, 85, 583-588. [CrossRef] [PubMed]

9. Ang, L.W.; Cutter, J.; James, L.; Goh, K.T. Seroepidemiology of Hepatitis B Virus Infection among Adults in Singapore: A 12-Year Review. Vaccine 2013, 32, 103-110. [CrossRef] [PubMed]

10. Liew, F.; Ang, L.W.; Cutter, J.; James, L.; Goh, K.T. Evaluation on the effectiveness of the national childhood immunisation programme in Singapore, 1982-2007. Ann. Acad. Med. Singap. 2010, 39, 532-541.

11. Alter, M.J. Epidemiology of Viral Hepatitis and HIV Co-Infection. J. Hepatol. 2006, 44, 6-9. [CrossRef] [PubMed]

12. Platt, L.; Easterbrook, P.; Gower, E.; McDonald, B.; Sabin, K.; McGowan, C.; Yanny, I.; Razavi, H.; Vickerman, P. Prevalence and Burden of HCV Co-Infection in People Living with HIV: A Global Systematic Review and Meta-Analysis. Lancet Infect. Dis. 2016, 16, 797-808. [CrossRef]

13. Lim, S.G. Time for Action on Viral Hepatitis. Ann. Acad. Med. Singap. 2016, 45, 27-30. [PubMed]

14. Update on the HIV/AIDS situation in Singapore 2017 (June 2018), MOH Resources \& Statistics. Available online: https://www.moh.gov.sg/resources-statistics/infectious-disease-statistics/hiv-stats/update-on-thehiv-aids-situation-in-singapore-2017-(june-2018) (accessed on 17 November 2018).

15. Lim, R.B.; Tan, M.T.; Young, B.; Lee, C.C.; Leo, Y.S.; Chua, A.; Ng, O.T. Risk factors and time-trends of cytomegalovirus (CMV), syphilis, toxoplasmosis and viral hepatitis infection and seroprevalence in human immunodeficiency virus (HIV) infected patients. Ann. Acad. Med. Singap. 2013, 42, 667-673.

16. Hyams, K.C. Risks of chronicity following acute hepatitis B virus infection: a review. Clin. Infect. Dis. 1995, 20, 992-1000. [CrossRef]

17. Fattovich, G.; Bortolotti, F.; Donato, F. Natural history of chronic hepatitis B: special emphasis on disease progression and prognostic factors. J. Hepatol. 2008, 48, 335-352. [CrossRef]

18. Alberti, A.; Chemello, L.; Benvegnù, L. Natural history of hepatitis C. J. Hepatol. 1999, 31, 17-24. [CrossRef]

19. Gerlach, J.T.; Diepolder, H.M.; Zachoval, R.; Gruener, N.H.; Jung, M.C.; Ulsenheimer, A.; Schraut, W.W.; Schirren, C.A.; Waechtler, M.; Backmund, M.; et al. Acute hepatitis C: High rate of both spontaneous and treatment-induced viral clearance. Gastroenterology 2003, 358, 80-88. [CrossRef]

20. Spradling, P.R.; Richardson, J.T.; Buchacz, K.; Moorman, A.C.; Brooks, J.T. Prevalence of Chronic Hepatitis B Virus Infection among Patients in the HIV Outpatient Study, 1996-2007. J. Viral. Hepat. 2010, 17, 879-886. [CrossRef] [PubMed]

21. Askari, A.; Hakimi, H.; Nasiri Ahmadabadi, B.; Hassanshahi, G.; Kazemi Arababadi, M. Prevalence of Hepatitis B Co-Infection among HIV Positive Patients: Narrative Review Article. Iran. J. Public Health 2014, 43, 705-712.

22. Chen, M.; Wong, W.-W.; Law, M.G.; Kiertiburanakul, S.; Yunihastuti, E.; Merati, T.P.; Lim, P.L.; Chaiwarith, R.; Phanuphak, P.; Lee, M.P.; et al. Hepatitis B and C Co-Infection in HIV Patients from the TREAT Asia HIV Observational Database: Analysis of Risk Factors and Survival. PLoS ONE 2016, 11, e0150512. [CrossRef] [PubMed]

23. Akhtar, A.; Khan, A.H.; Sulaiman, S.A.S.; Soo, C.T.; Khan, K. HBV and HIV Co-Infection: Prevalence and Clinical Outcomes in Tertiary Care Hospital Malaysia. J. Med. Virol. 2016, 88, 455-460. [CrossRef]

24. Utsumi, T.; Lusida, M.I. Viral Hepatitis and Human Immunodeficiency Virus Co-Infections in Asia. World J. Virol. 2015, 4, 96-104. [CrossRef] [PubMed]

25. Martinello, M.; Amin, J.; Matthews, G.V.; Dore, G.J. Prevalence and Disease Burden of HCV Coinfection in HIV Cohorts in the Asia Pacific Region: A Systematic Review and Meta-Analysis. AIDS Rev. 2016, 18, 69-80.

26. Huy, B.V.; Vernavong, K.; Kính, N.V. HBV and HCV Coinfection among HIV/AIDS Patients in the National Hospital of Tropical Diseases, Vietnam. AIDS Res. Treat. 2014, 2014, 581021. [CrossRef]

27. Zhou, Y.H.; Liu, F.L.; Yao, Z.H.; Duo, L.; Li, H.; Sun, Y.; Zheng, Y.T. Comparison of HIV-, HBV-, HCV- and Co-Infection Prevalence between Chinese and Burmese Intravenous Drug Users of the China-Myanmar Border Region. PLoS ONE 2011, 6, e16349. [CrossRef]

28. Goh, K.T.; Doraisingham, S.; Tan, K.L.; Oon, C.J.; Ho, M.L.; Chen, A.J.; Chan, S.H. The Hepatitis B Immunization Programme in Singapore. Bull. World Health Organ. 1989, 67, 65-70.

29. Lee, C.E. Tackling Subutex Abuse in Singapore. Singap. Med. J. 2006, 47, 919-921. 
30. Overview of Singapore's Drug Situation in 2017, Central Narcotics Bureau. Available online: https://www.cnb.gov.sg/docs/default-source/drug-situation-report-documents/cnb-annual-statsrelease-for-2017_12-jun.pdf (accessed on 17 November 2018).

31. Soh, B.Y.-M.; Kumar, R.; Ekstrom, V.S.-M.; Lin, C.Y.-H.; Thangaraju, S.D.; Tan, H.H.; Chan, K.P.; Choong, L.H.L.; Teo, D.; Chow, W.C. Prevalence of Hepatitis C Virus Infection and the IL28B Genotype Polymorphism among Blood Donors and High-Risk Populations. Singap. Med. J. 2018, 60, 34-39. [CrossRef]

32. Gower, E.; Estes, C.; Blach, S.; Razavi-Shearer, K.; Razavi, H. Global Epidemiology and Genotype Distribution of the Hepatitis C Virus Infection. J. Hepatol. 2014, 61, S45-S57. [CrossRef] [PubMed]

33. Panel on Antiretroviral Guidelines for Adults and Adolescents. Guidelines for the Use of Antiretroviral Agents in Adults and Adolescents Living with HIV. Department of Health and Human Services. Available online: http: //aidsinfo.nih.gov/contentfiles/lvguidelines/AdultandAdolescentGL.pdf (accessed on 17 November 2018).

34. Peters, L.; Klein, M.B. Epidemiology of Hepatitis C Virus in HIV-Infected Patients. Curr. Opin. HIV AIDS 2015, 10, 297-302. [CrossRef] [PubMed]

35. Kaplan, E.; Heimer, R. HIV Prevalence among Intravenous Drug Users: Model-based Estimates from New Haven's Legal Needle Exchange. J. Acquir. Immune Defic. Syndr. 1992, 5, 163-169.

36. Van Den Berg, C.; Smit, C.; Van Brussel, G.; Coutinho, R.; Prins, M. Full Participation in Harm Reduction Programmes Is Associated with Decreased Risk for Human Immunodeficiency Virus and Hepatitis C Virus: Evidence from the Amsterdam Cohort Studies among Drug Users. Addiction 2007, 102, 1454-1462. [CrossRef]

37. Hagan, H.; Jarlais, D.C.; Friedman, S.R.; Purchase, D.; Alter, M.J. Reduced Risk of Hepatitis B and Hepatitis C among Injection Drug Users in the Tacoma Syringe Exchange Program. Am. J. Public Health 1995, 85, 1531-1537. [CrossRef] [PubMed]

38. Singh, P.S.J.; Azman, A.; Samsurijan, M.S.; Badaruddin, R.F.R.; Vadevelu, K.; Yahaya, M.H.; Latiff, A.R.A. Implementation Dilemmas of the Needle Syringe Exchange Programme (NSEP): Between the Law and Prevention. Pac. Sci. Rev. B Humanit. Soc. Sci. 2016, 2, 53-57. [CrossRef]

39. Lim, S.G.; Lee, G.H. Pathway to Hepatitis Elimination and Control. Ann. Acad. Med. Singap. 2018, 47, 435-437. [PubMed]

(C) 2019 by the authors. Licensee MDPI, Basel, Switzerland. This article is an open access article distributed under the terms and conditions of the Creative Commons Attribution (CC BY) license (http://creativecommons.org/licenses/by/4.0/). 